\title{
LOS NOMBRES DE ENCLAVES DE LOS PAÍSES NÓRDICOS EN EL “LIBRO DEL CONOSCIMIENTO DE REYNOS, TIERRAS Y SEÑORIOS" (S. XIV) Y EN UNA RELACIÓN DEL DIPLOMÁTICO IUAN SCHEFFER (1627). UNA COMPARACIÓN DIACRÓNICA
}

\author{
Jorge Simon Izquierdo Díaz \\ Doctorando, UNED \\ jsid12@yahoo.dk
}

RESUMEN: La comparación diacrónica entre el léxico empleado en el Libro del conoscimiento de reynos, tierras y señoríos ${ }^{1}$, de autor anónimo, siglo XIV, y una relación del diplomático luan Scheffer ${ }^{2}$ (1627) ilustra el grado de conocimiento de la costa de los países nórdicos en nuestro país, escaso hasta la Edad Moderna y de rápido crecimiento desde el siglo XVII.

Palabras clave: Literatura y documentación de viajes, costa de Escandinavia, Libro del conoscimiento de reynos, tierras y señoríos, luan Scheffer, diacronía y cambio léxico.

\section{PLACE NAMES IN NORTHERN COUNTRIES IN “LIBRO DEL CONOSCIMIENTO DE REYNOS, TIERRAS Y SEÑORIOS" (14TH CENTURY) AND IN A OFFICIAL LETTER BY DIPLOMAT IUAN SCHEFFER (1627). A DIACHRONIC COMPARISON}

1. Año de edición y autor son desconocidos. En este trabajo voy a utilizar la edición de Marcos Jiménez de la Espada: Anónimo: Libro del Conoscimento de reynos, tierras y señoríos. Ed. Marcos Jiménez de la Espada. Sevilla. 1874.

2. Iuan Scheffer: Relación cierta i verdadera, sacada de avisos una y otra vez confirmados, de la rota $i$ huida del Rei de Denemarca, i total destrucció de su campo y exercito, i señaladas vittorias que la Sacra Magestad Cesare ha tenido aora nuevamente de sde 18 de octubre hasta fin de noviembre de 1627 en los Paises de la Saxonia inferior, i tierra firme del Septentrion. 
ABSTRACT: The lexical comparison between the vocabulary used in the anonymous Libro del conoscimiento de reynos, tierras y señoríos, $14^{\text {th }}$ century, and a official letter by diplomat luan Scheffer (1627) illustrates the change of knowledge of the coast of the Nordic countries in our country, which was scarce until the Modern age, and it rapidly increased during the $17^{\text {th }}$ century.

Keywords: Travel literature and documents, Northern Europe's coastal geography, Libro del conoscimiento de reynos, tierras y señoríos, luan Scheffer, diachronic lexical change.

Recibido: 8 de Junio de 2017 Aceptado: 14 de Diciembre 2017

\section{El Libro del conoscimiento de reynos, tierras y señorios}

\section{Descripción del libro}

El Libro del conoscimiento es dos cosas: un relato de viajes, posiblemente imaginarios ${ }^{3}$, y un memorial o repertorio heráldico mundial del siglo XIV.

El origen del libro es problemático en diversos sentidos. Se desconocen autoría y fuentes. Es un libro de autor anónimo, y se discute la procedencia del autor, si era de origen castellano o aragonés ${ }^{4}$ y la naturaleza del viaje, ya que se duda de que el autor haya realizado tal viaje.

Se data en el siglo XIV, probablemente entre 1350 y 1400, posterior a 1375 si se considera el Atlas catalán como su fuente principal (Marino 1999). El autor pudo seguir un mapamundi del siglo XIV - pero se desconoce cuál (Lacarra 1999).

No quedan originales del XIV, y los cuatro originales que se conservan son del siglo XV (Marino 1999): 2 en la Biblioteca Nacional de Madrid (códices $S$ y N), 1 en la Universidad de Salamanca (Códice R), 1 en la Bayerische Staatsbibliotek (Códice Z). Las copias originales S, N y R son castellanas y la Z es aragonesa, esta última con omisiones, es posterior y menos creíble. Las ediciones de Jiménez de la Espada (1874) y Nancy Marino (1999) están basadas en

3. Un debate similar ofrece el mapa de los hermanos Zeno, de finales de 1380, publicado en 1558 en Venecia. Sus defensores, entre ellos A.E. Nordenskjöld (Om bröderna Zenos resar och de äldsta kartor öfver Norden, de 1883), creen que es cierto que los hermanos Zeno viajaron al norte de Europa, lo que sus detractores, por ej. C.C. Zahrtmann, niegan. La historiografía contemporánea lo considera falso, ya que sus datos proceden de Claudius Clavus (Kjelbo 1966).

4. El autor dice al principio del libro que nació el 11 de septiembre de 1305, en Castilla, durante el reinado de Fernando IV. Jiménez de la Espada añade que es un monje franciscano, aunque faltan referencias para tal afirmación (Marino 1999). 
el manuscrito S, y la de Lacarra y Montaner (1999) en el manuscrito Z. Según Lacarra y Montaner (1999) el manuscrito Z es una copia aragonesa del XV de un códice castellano perdido, destinado a algún potentado, posiblemente Juan II (1406-1454). ${ }^{5}$

El Libro es más bien un relato imaginario, pero con una finalidad didácticodoctrinal $^{6}$, y en el que el autor repasa la geografía y política del mundo conocido. Para la crítica es un viaje ficticio, total o en parte, que entronca con la literatura de viajes desde la experiencia personal del autor 'viajero', "que sigue un itinerario, aunque sin especificar tiempo" (Martín de Riquer 1978). No se descarta que, para el caso de Europa, haya realizado alguno de los viajes que describe porque el itinerario por Europa es materialmente factible, por tierra y por mar, incluso en diferentes etapas de su vida, aunque la cantidad de datos resta credibilidad al relato (Marino 1999). El libro sirvió de guía de viajes para viajeros posteriores (las expediciones de Bethencourt a Islas Canarias y Guinea, y de Enrique el Navegante a la costa de Africa), y de inspiración para libros de texto de geografía y heráldica (Marino 1999).

Los recorridos por Europa son descritos como viajes de ida y vuelta. El punto de partida y llegada es Sevilla. De la península ibérica viaja a Centroeuropa y de Colonia hacia Holanda, Flandes y Dinamarca, bordea la costa sur del Báltico, baja hasta Rumania y Ucrania. El recorrido salta a Suecia, de ahí a Noruega, se embarca hacia Dinamarca, regresa a Noruega, de donde parte para las islas británicas y regresa a España haciendo escala en las islas Feroe e Islandia.

\section{La cosmografía del Norte de Europa en la Edad Media}

Griegos y romanos tenían poco conocimiento de las tierras más allá del Estrecho de Gibraltar. Hay que remontarse a los griegos Pytheas de Marsilia (hacia el siglo IV a.C.), con la conocida referencia a la isla de Thule (¿Islandia?) y al ámbar de las costas del Báltico, el Sinus codanus del romano Plinio el Viejo (s. I d.C. ), y Ptolomeo (s. II d. C.), cuya geografía original se ha perdido, para encontrar las primeras referencias a la costa escandinava en la cosmografía europea ${ }^{7}$. Ptolomeo no dibuja, da coordenadas. Su geografía, bastante ajustada para el caso danés, incluye la península címbrica (Jutlandia) - de la desembocadura del rio Elba a Ska-

5. El análisis paleográfico y lingüístico realizado por Lacarra y Montaner revela que la letra gótica del manuscrito $Z$ tiene rasgos del siglo XVI. Se trata de letra gótica híbrida aragonesa con bastarda aragonesa, diferente a la cursiva castellana. El manuscrito $Z$ abunda en aragonesismos: nasal $\tilde{n}>$ ny, yn, líquida palatal: II $>$ ly, velar oclusiva sorda: $k>q u$, velar oclusiva sonora: gu > go (Lacarra y Montaner 1999).

6. Para la literatura medieval con fines didácticos, consúltese Rafael Segura (2004: 538).

7. Los periplos griegos, itinerarios dibujados a modo de libros de viajes sobre las rutas comerciales, ayudaban a los navegantes con información sobre vientos, corrientes, y accidentes geográficos. 
gen calcula que hay 13 grados de longitud, hoy sabemos que hay 7 -, limitada por una serie de islas (las actuales Anholdt, Læsø) y puntos de la costa (los actuales Vendsyssel, Thy y Mors) al oeste y tres islas grandes al este (las actuales Fiona, Selandia y Loland- Falster). Sin embargo Ptolomeo desconoce los territorios de Noruega y Suecia, ya que la península escandinava (Scandia) es descrita como una isla al norte de los límites de Europa ${ }^{8}$. Las denominaciones romanas combinan criterios político geográficos (Germania, Sarmatia, Codannia, Thule, Scythae) con un criterio étnico (cimbrios, teutones, escitas, hiperbóreos), como hace Pomponio Mela (s. I d.C.), o climáticos, como el mapa de Macrobio (s. IV), que incluye el norte de Europa dentro de la zona frígida (Barber 2005). ${ }^{9}$

En la Edad Media, la literatura de viajes por autores nórdicos describe dos rutas atlánticas. La una, es la navegación entre las costas del Atlántico norte, de Noruega a Groenlandia, descrita por primera vez en la saga de Olof Tryggvesson, hacia 1200. La otra es la ruta peregrina hacia el sur incluida en la Adamus Gesta Hammaburgensis ecclesiae pontificum o crónica de Adam, que describe la ruta peregrina de 35 días de Ribe a Siria, con escala en Ferrol. (Dahlgren 1897). La crónica de Adam es el principal documento medieval para conocer la geografía del norte de Europa durante la transición al cristianismo.

El Jordebog (Libro del censo) del rey danés Valdemar (siglo XIII) incluye una descripción de nombres y lugares de Dinamarca -que entonces incluía el Sur de la actual Suecia- muchos latinizados (Dahlgren 1897). El primer mapa de Dinamarca, esbozando el sur de la península escandinava, es el mapamundi de Ibn Idrissi (s. XI), incluido en la Tabla rogeriana para la corte siciliana del normando Roger II. Sus fuentes son Paulo Orosio y Ptolomeo (Lassen y Madsen 1981). El primer mapa completo de Escandinavia es de Claudius Clavus, en 1427, incluida en la edición de Ptolomeo de Bolonia, en 1477. Clavus nació en la isla de Fiona en 1388. Su mapa consiste en un círculo dividido en tres partes: Danorum regi, Suetia regi y Noruegia regi, y distingue entre Jutlandia y Fiona (Bramsen 1997).

En su traducción de Paulo Orosio (s. V) al sajón, el rey inglés Alfredo el Grande (s. IX) intercala tres capítulos dedicados al norte de Europa: uno sobre Alemania, y los otros dos son, uno, los viajes por el norte de Europa de Othere de Hålogaland (contemporáneo de Alfredo) y el otro el viaje de Wulfstan (Jørgensen 1983). Alemania es descrita a través de accidentes naturales y grupos étnicos, y los datos son más inseguros cuanto más septentrional es la región descrita.

El viaje de Othere cita 22 puntos geográficos de islas y ciudades en la ruta entre Sciringshæl (Noruega) y Hedeby o Haedun, en la actual Alemania. Othere

8. De acuerdo al mapa de Claudius Clavus (s. XV), que reproduce la Escandinavia descrita por Ptolomeo.

9. El mapa circular de Macrobio (s. IV d.C.) describe 4 continentes y 5 zonas climáticas. 
invierte las posiciones geográficas de las regiones del estrecho del Kattegat entre Suecia y Dinamarca: denomina Gotland a Jutlandia y Denameacr a Scania.

De todas maneras, la cartografía nórdica es marginal a los mapas medievales de $\mathrm{T}$ en $\mathrm{O}^{10}$ y a los portulanos mediterráneos. En la representación teológica medieval del mundo habitado e inhabitado desde un centro inamovible, las costas de Escandinavia, fuera de las rutas comerciales o periplos mediterráneos, aparecen vagamente perfiladas, y en función del centro de interés, que es Alemania y las rutas de la Hansa. Los mapas en T en O de Beato de Liébana (siglo VIII), Ebstorf (siglo XIII, Alemania) y Haldingham (siglo XIII, Inglaterra), que describen los tres continentes conocidos (Asia, Europa y África) relegan a Escandinavia a un lugar marginal, en la parte inferior izquierda del mapa (Nordenskjöld 1897).

El Libro del conocimiento menciona las ciudades de Ribe y Tønder, pero no Hedeby, importante enclave vikingo, lo que me lleva a pensar que las fuentes del autor anónimo son más bien los portulanos medievales. En los portulanos del siglo XIV el Mediterráneo sigue siendo el centro de orientación, pero a diferencia de los mapas en $\mathrm{T}-\mathrm{O}$, orientados hacia el Este, con Jerusalén como capital del mundo, los portulanos ya están orientados hacia el norte. Incluyen rosa de los vientos, y viñetas de ciudades, escudos de armas y banderas (Barber 2005). Los portulanos incluyen nueva información para los navegantes, integrando los conocimientos de las cruzadas, los peregrinos y la expansión del comercio por el Mediterráneo (Kjelbo 1966). La adecuación topográfica es escasa, más interesados los autores en describir las rutas comerciales entre las costas del Mediterráneo, el mar Negro y la costa atlántica hasta Frisia. Van ampliando su ámbito de oeste a este, integrando muevas tierras.

A continuación se describen portulanos del XIV ${ }^{11}$ que pudieron servir de inspiración al anónimo autor del Libro del conoscimiento. La cartografía de Sanudo-Vesconte es de las primeras décadas del siglo XIV. En el portulano, de hacia 1310-1330, se dibuja Noruega como un largo brazo de tierra, Suecia es dibujada con tres círculos (Suecia, Alandia, Finlandia), en el mar Báltico o mar del Este (Östersjön) se incluyen las islas de Ossilia y Gotlandia. Dinamarca es dibujada como la península címbrica, dentro de la cual el autor ha escrito los nombres de Dania y Jutia. Hacia el oeste, en mitad del océano, aparece la isla de Ybernia, que puede ser Islandia. En el mapamundi de 1320 aparecen Suecia, Noruega y Frisia, pero no el nombre de Dinamarca. El portulano de Angelino Dalorto, de 1325, da nombres de poblaciones costeras pero confunde el nombre de Skandia con la isla de Selandia ${ }^{12}$. La costa de Dinamarca y la del sur de

10. Mapa de T en O: un tipo de mapamundi realizado en la Edad Media, que representa las tres partes del mundo conocido (Asia, Europa y Africa) como una $\mathrm{T}$ inscrita en una $\mathrm{O}$. También se conoce como mapa Orbis Terrarum.

11. A.E. Nordenskjöld (1897) ofrece una descripción con copias de los portulanos medievales.

12. Probablemente debido a la similitud entre los nombres Skandia y Selandia. 
Suecia (Gothland) aparecen en el portulano de G. da Carignano (1327), editado en Florencia, y destruido durante la segunda guerra mundial. Angelino Dalorto realizó otro portulano en 1339 en el que distingue entre Dinamarca, Suecia y Noruega. En el primer país incluye a Jutlandia y las islas de Salandia, Finonia y Langland, a las que ubica erróneamente al norte de Jutlandia. Utiliza el motivo de las banderas, que alza sobre mástiles instalados en sendos castiIlos circulares: Dinamarca con tres leones, Suecia, con dos leones y Noruega, con un león. El portulano Laurenziano-Gaddiano, de 1351 incluye Jutlandia y Báltico y la costa sur de Noruega y el Báltico. La fuente más probable para el Libro del conoscimiento, por la cercanía, quizá sea el Atlas mallorquín de la familia Cresques, de 1375.

El Libro del conoscimiento repite la clásica descripción de los rasgos geográficos del norte de Europa como tierra deshabitada, con días de 6 meses y noches de 6 meses. Dinamarca es una zona de frontera que marca el punto de inicio del portulano de la costa europea occidental, que va de Dinamarca (Jutlandia) a Marruecos. Dinamarca es una prolongación de Flandes, y va adquiriendo identidad propia, Suecia y Noruega son terra incógnita, cuya población el autor anónimo del libro describe mediante fabulaciones -"ay ende vnas gentes que han las cabecas fincadas en los pechos que non ha cuello njnguno" (Lacarra 1999)- quedando la duda de si realmente el autor ha estado o no allí, cuando dice: "pero yo no las vi" (Pg. $5 \mathrm{v}$ en la ed. de Lacarra). ${ }^{13}$

\section{Los armoriales}

La originalidad del Libro del conoscimiento reside en apuntar el armorial en el libro de viajes (Muntaner 1999). Al modo de los portulanos que le sirven de inspiración, que estaban decorados profusamente con banderas, ciudades, tapices, miniaturas y leyendas, sintetizando información para los navegantes, el Libro del conoscimiento contiene un extenso repertorio de señales et armas - banderas y armorial blasonado. El autor pudo utilizar como fuente los armoriales europeos, que ya aparecen en el siglo XIII, por ej. el Armorial Wijnbergen (1270-85) (Lacarra 1999).

13. La descripción de Noruega en el portulano de Cresques ilustra los prejuicios acerca de la región, considerada salvaje e inhóspita: "Aquesta regió de Nuruega es molt aspera, e molt Freda e montanyosa, salvagossa et plena de boschs, los habitadors de la qual més viuen de peix e de caca que de pa, avena s'hi fa fort pochs per lo gran fred; moltes feres i ha, co es cervos, orsos blanchs e grifalts". Rasgos salvajes, tan distintos del cálido, hogareño y civilizado Mediterráneo, y donde no se come pan porque el clima no permite crecer la avena. 
El sentido de las armerías es identificar territorios soberanos y señoríos. El Libro del conoscimiento es una fuente capital para conocer las banderas y heráldica europea del siglo XIV (Martín de Riquer 1987).

En cuanto a la región nórdica, Dinamarca aparece dividida en 2 regiones con sendos blasones: el blasón de Dacia (Jutlandia), un pendón de oro con tres leones prietos, y el blasón de Selandia, que aparece unida políticamente a Noruega. El autor le da preeminencia a Noruega, y Selandia queda reducida a isla. Suecia está dividida entre el reino de las islas Visvy y Oxilia, y lleva por señales un pendón de bandas amarillas y cadenas atal, y el reino de Gocia, cuya señal es un pendón atal (es decir, satinado). ${ }^{14}$

Léxico de lugares de Escandinavia en Libro del Conoscimento de reynos, tierras y señoríos

El Libro del Conoscimiento manifiesta tendencias gramaticales y fónicas que configuran los rasgos idiomáticos que marcan el paso de la lengua medieval a la moderna.

El autor vacila en el uso de la consonante inicial fricativa en el término geográfico solanda (alveolar), al que más tarde llama colanda (velar) (manuscritos S y Z), pero no está claro si se refiere al país de Holanda o a la isla danesa de Selandia.

A pesar de tratarse de un libro de viajes, la falta de descripción de escenas de vida de los habitantes de los países por donde pasa hace que la prosa del autor franciscano resulte monótona y esquemática para el lector. Sirva de ejemplo la descripción de su ruta por el golfo de Frisia "en el qual golfo son quatro yslas a la vna dizen Ruyna a la otra dizen erria a la otra finonia A la otra dizen glangante el Rey de frisa a por señales vn pendon de oro con tres leones prietos luengos atales" (Manuscrito S, p. 10 en Jiménez de la Espada, 1875).

De estos topónimos nórdicos el más reconocible por un hispanohablante actual quizás sea finonia que podría corresponder tanto a la isla de Fanø, en la costa oeste de Jutlandia, frente a la actual Esbjerg, como a la isla de Fionia (Fyn), Finnonya en Dalorno, Fininia en el atlas catalán, en el centro del país, y cuya capital es Odense. Fiona es una isla principal, pero por proximidad geográfica bien pudiera el autor referirse a Fanø, que se encuentra a continuación de Frisia, y por la traducción común de la palabra danesa ' $\varnothing$ ', que quiere decir isla, como sufijo 'ia'. De este manera se puede entender que haya traducido la isla de Rom (Romø) como Ruyna (aquí creando un diptongo o>uy) quizá por metátesis.

La isla de Erria podría corresponder a la isla danesa de Arø (Ærræ en el censo del rey Valdemar) y ganglante (Janglant en el manuscrito de Lacarra) puede ser

14. Para la concordancia de armoriales, véase Lacarra (1999). 
Helgoland, ciudad de la costa noroeste del actual Slesvig (Alemania) o la isla de Langæland (censo del rey Valdemar). El autor extiende el castellanismo cuando se trata de traducir un extranjerismo, tal como lo ilustra la terminación -lante en ganglante. Land quiere decir país, tierra y el autor lo castellaniza añadiendo una -e final, quizás influido por la tendencia lingüística del siglo XIV a restablecer la e final de palabra (por ejemplo: fiz > fize; véase Lapesa 1942, p. 136).

El autor identifica dacia con el reino de Dinamarca, ya que en la Edad Media se utiliza Dacia para denominar a Dinamarca"15, ("Partime del Reyno de frisa e entre luego en el reyno de dacia"). Se mantiene la grafía c cedilla (ç) medieval para expresar la fricativa dental sorda /c/ ante /i/ en el nombre de país Daçia (Dania/Dacia en Ptolomeo, Dacie en el censo del rey Valdemar, Dasia en el atlas catalán, Dacia en castellano actual).

Por otra parte, no respeta la mayúscula inicial del nombre propio del país: escribe frisia, dacia, y la misma norma ortográfica sigue para escribir el nombre de las ciudades por donde pasa: viborg, burgalensis, etc. Sin embargo vacila en el empleo de la consonante mayúscula en unas pocas palabras: escribe $R u$ menia, pero noruega; escribe Regis, pero tronde, una inseguridad propia de un período de transición hacia la consolidación de la norma ortográfica tal como hoy la conocemos.

El autor no usa apócope, lo que coincide con la tendencia general del periodo a la eliminación de la misma (véase Abad Nebot 2008).

La importancia de Alemania le lleva a nombrar como 'El mar de alemaña' al Báltico, que en lengua germano-escandinava contemporánea es el mar del Este (Øster sø, en danés, Östersjön, en sueco, Ostsee en alemán). El cartógrafo sueco Olaus Magnus, autor del primer mapa de Escandinavia, en el siglo XV, no utiliza un término común para todo este gran mar interior pero lo divide en tres zonas geográficas, que nombradas de oeste a este son: mare Goticum (entre la isla de Gotland y Jutlandia), mare Livoicum (la costa de Livonia, que corresponde a las actuales Estonia, Letonia y Lituania) y el mare Sveticum - el actual golfo de Botnia entre Suecia y Finlandia.

El recorrido del autor viajero es un recuento de países y principales ciudades. Tras presentar el 'mar de alemaña' el autor pasa a describir la ubicación de las 12 ciudades principales del Reyno de Dacia y que comienza en la actual Viborg, denominada burbena (manuscritos S y Z), Wiberg en la crónica de Adam de Bremen (s. XI), Viborg, su nombre actual, en el censo del rey Valdemar, Vuijller en Dalorto (1339) y Viber en el atlas catalán, situada al norte de la actual Jutlandia y capital histórica de la península de Jutlandia. Burgalensis (Burgalenssis en el manuscrito Z) es Børglum, Borlunheret en el censo del rey Valdemar y

15. No confundir con la provincia romana de Dacia, que corresponde con la actual Rumanía. 
Burgalensis en Dalorto. El enclave de biua (manuscrito S) o vibe (Manuscrito Z) es Ribe, la Ripe de la crónica de Adam, Ripæ en el censo de Valdemar, Rip Ripes en Dalorto, Ripiss en el Atlas catalán y Portus Ripae en el portulano Laurentziano. Ribe es la ciudad portuaria más antigua de Dinamarca, fundada hacia el año 800. El enclave de abenbruc es, según Jiménez de la Espada la actual Abenbrök en Holstein, norte de Alemania, Ilamada Abenbrut en el manuscrito Z (Lacarra) y Operom en el portulano de Dalorno, pero, en mi opinión, pudiera tratarse de la actual Åbenrå en Jutlandia del sur. El enclave siguiente es tandeuz (la ciudad de Randers, en Jutlandia), Tandens en el manuscrito Z (Lacarra), Rondus en el censo del rey Valdemar, Randers en Dalorto (1339). La actual capital de Jutlandia, Århus, es denominada artuz, que se asemeja al nombre en danés antiguo aros (rey Valdemar), y Arus en Dalorto, pero Arhusan en Adam, y que hoy día se pronuncia Jhus, con $\mathrm{h}$ aspirada. El manuscrito $\mathrm{Z}$ la llama arbis, ya bastante alejado. La ciudad de orens es la actual Odense, Oresnes en el manuscrito $Z$, es Odensve en Adam. El nombre de ardonxep , Anderxep en el manuscrito Z, puede corresponder a Haderslev, al sur de Jutlandia, denominada Hathærslefheret en el censo del rey Valdemar. El enclave de corp corresponde a Gottorp, en Slesvig, Gocop en el manuscrito Z, y dandora, es Tønder, la Tundær en el censo del rey Valdemar. Otros puntos geográficos son damesmare (sin traducción, posiblemente el mar danés), y dasia, bonia o Dacia.

Hay que hacer notar la tendencia del autor a ajustar los topónimos al sonido en castellano por medio de la grafía. Excepción hecha de Borlum (Børglum), que traduce como burgalensis (el burgo), en antiguo nórdico burgh es fortaleza, el resto de las traducciones traiciona la palabra original: $\mathrm{o}>\mathrm{u}$ en Abenbrok; $r>t$ en Randers, además de la vocalización del binomio consonántico rs, ajeno al uso castellano; $h$ aspirada > t en Århus, $d$ > r en Odense; g > c en Gottorp, y eliminación de consonante intervocálica t. Vacila en el empleo de las terminaciones en vocal: elimina la -e final de Odense (orens), pero la añade en Donder (dandora), con ánimo castellanizante.

El texto abunda en confusiones geográficas, por lo que se pone en duda que el autor haya realizado realmente tamaño viaje por Europa del Norte. En "E desta punta dacia fasta noruega son sesenta millas de trauiesa e el Rey desta dacia a por señales vn pendon de oro con tres leones prietos atales" no queda claro si se trata de una punta o cabo de la región de Slesvig-Holstein, desde el que mide la distancia de las 60 millas hacia el norte ó noreste. La descripción del pendón de oro con tres leones podría corresponder al escudo de Dinamarca (que data de 1270), por lo que el autor confunde Noruega con Dinamarca (que en el siglo $\mathrm{XV}$ son parte del mismo reino). Es posible que el texto se base en un antiguo cartulario - los comentaristas apuestan por el Atlas catalán de 1375.

Nuestro autor sigue su periplo hacia Sajonia (escribe Xaxonia), pasando por las ciudades de lubec (Lubeck), rosgot (Rostock), bordeando el Báltico hasta la ciudad de dansicha (Danzig), en Polonia, y en cuyo golfo desemboca el río Vístula, que el autor llama turonie. Más al sur, de camino a boemia (Bohemia) 
ubica avandalia, la tierra de los vándalos, cerca del rio Alba (Elba), y de cuyo topónimo deriva el nombre de Andalucía, asegura el mismo.

En este párrafo nos encontramos con fenómenos similares a los ya descritos, con la castellanización en lubec y dansicha y la metátesis en rosgot (Rostock). Turonie es arcaísmo. Destaca el doble empleo inicial e intervocálico de la consonante $/ \check{s} /$, diferente del empleo en el siglo XVII, donde, tras un periodo de asimilación a la interior, la inicial /š/ se ha estabilizado en S (por ejemplo, en 'Duque Francisco Carlos de Saxonia', uno de los protagonistas del escrito de luan Scheffer).

El autor anónimo utiliza los términos de geografía castellanos: cibdat, prouincia, isla,- con la inseguridad de los mismos, por ejemplo escribe perrocHias (parroquias). El autor utiliza el término cib'dat. La palabra latina Civitate dio cibdat. Aunque su uso es considerado como decadente (Lapesa 1942), el autor conserva el grupo consonante ( $\left.b^{\prime} d\right)$, un momento fricativo exagerado (Abad Nebot 2008) que prácticamente se vocaliza en el habla cotidiana para dar lugar a la palabra 'ciudad' del español moderno.

Tras pasar por boemia (Bohemia) y la prouincia de Rumenia (Rumanía) el autor regresa al norte para describir Suecia, "e entra por vna grand prouincia que dizen sueia". Empieza por roderin (Roggerwick), tarsa (Skarsta), andine (sur de Suecia), chicobergis (Wexio), landis (Lund), ystat (Ystad), formeans (Soderman). Hace referencia a dos lagos principales del país: Escarse, que podría ser el lago de Wener, que baña la ciudad de Skar ('Skar se' ó Skar sö, lago de Skar) y Estocol (Estocolmo), que podría corresponder al lago Malärn, que empieza unos unos kilómetros al oeste de la ciudad.

Prosigue su viaje hacia la costa este, cruza en barco hasta la isla de gotlandia (Gotland) (castellanizada) y regresa a la península, donde recorre la región que hoy se conoce como Östgotland (Gotia del Este), "Party de gotlandia e torneme para gotia vna Prouincia ques entre sueuia e noruega", una región en la que se localizan tres ciudades importantes: estocol (Estocolmo), calman (Kalmar) y surdepinche (Sonderköping). Identifica esta Gotia con la patria de los visigodos. El empleo de recursos lingüísticos como la conjunción ' $\mathrm{e}$ ' y la repetición de palabras, que funcionan como conectores del discurso (Santiago, 2004, p. 537) tienen un antecedente en 'El Libro del caballero Zifar' (novela de caballerías de 1305 que, como indica Abad Nebot, marca el genuino inicio del género en nuestro país).

El autor desconoce la existencia de Finlandia, y cree que el Báltico está bordeado por Alemania y Polonia (Palonia) por el sur y las "sierras de noruega" por el norte. Tras pasar por Östgotland abandona por tanto el rumbo noreste y se dirige al norte, a Noruega, un país que asocia a cuatro ciudades importantes; tres en la costa oeste: "la que dizen Regis" (Bergen) -la llama Regis porque es la sede de la coronación real-, nidroxia (Nídaros), tronde (Trondhjem)-creo que 
es una confusión, porque Nídaros y Trondhjem son la misma ciudad- y trunberec (Tonsberg), al sur.

El autor afirma que su partida de Noruega se produce en una nave de ingleses en dirección a la isla de cola ('insola cola') (Öland) para arribar a la isla de salanda (Selandia), pasando por lister (Listerby, cerca de Karlskrona, costa este de Suecia) y bondola (Bornholm, isla danesa en el Báltico); sin embargo ha cometido un error en su descripción porque Öland se encuentra en el Báltico. Las referencias a ciudades de la isla de Selandia se centran en salandi (la capital del país, Copenhague), risent (Ringsted), escondin (Stor Hedding), alenda (Lolland ó Lolandia, isla al sur de Selandia). Pone rumbo a Escocia, es de suponer que cruzando el Øresund, y hace escala en tille (Thysted, en el norte de Jutlandia, o incluso la región de Telemark, en el sur de Noruega), para llegar a Donfres (Dunfries), ya en las islas Británicas.

Para concluir, los topónimos nórdicos parecen estar traducidos de manera sui géneris. El autor castellaniza los términos extranjeros en un intento de hacer corresponder la ortografía castellana a lo que realmente se pronuncia, pero parece echar de menos una norma ortográfica que seguir.

\section{Texto diplomático de Iuan Scheffer}

El segundo texto es luan Scheffer: Relación cierta $i$ verdadera, sacada de avisos una y otra vez confirmados, de la rota i huida del Rei de Denemarca, i total destrucció de su campo y exercito, i señaladas vittorias que la Sacra Magestad Cesare ha tenido aora nuevamente de sde 18 de octubre hasta fin de noviembre de 1627 en los Paises de la Saxonia inferior, i tierra firme del Septentrion. (S. XVII)

\section{La cartografía nórdica en la Edad Moderna}

La cartografía nórdica se desarrolla entre los siglos $\mathrm{XV}$, impulsada por el redescubrimiento hacia 1400 de la cartografía de Ptolomeo, publicada en Florencia en 1406, y XVI, al abrigo de la tradición de cartografía neerlandesa de Ortelius y Mercator (Kejlbo 1966). Se van incorporando países y regiones de la costa nórdica, que va poco a poco integrándose en la cartografía europea: Thule, Scandia, las islas menores entre Scandia y Jutlandia, la terra incógnita más allá de Noruega, que cierra el mapamundi por el norte (Alonso y Zapatero 2000).

Desde mediados del siglo XVI, beneficiándose de los proyectos de las compañías de navegación hacia Asia, la costa nórdica se perfila cada vez más claramente. En 1532 Marcus Jordan imprime el primer mapa detallado de Dinamarca, reimpreso en el Theatrum Orbis Terrarum de Abraham Ortelius, Amsterdam, 1570 (Bramsen 1997). 
La Carta Marina del dominico sueco Olaus Magnus es de 1525, y en 1568 se imprime el primer mapa marítimo hecho en Dinamarca, Søkortet offner Øster oc Vester Søen ('Carta marina sobre los mares del este y del oeste'), por Lauritz Benedict (Kjelbo 1966). El Spieghel der Zeevaerdt ('Espejo de navegación') de Lucas Janz Zoon Waghenaer, el primer portulano nórdico, es de 1584. La costa escandinava aparece perfilada en los mapas de Bartolomeo Lasso, hacia 1590, y en el mapamundi de Petrus Plancius (1590) (Martín-Merás 2000). La versión cartográfica de Lapponiae, Bothniae, Cajanieaeque, Regni Sueciae Provinciarum Septentrionalium nova deliniatio, sculta anno Domini 1611 de Anders Bureo es de 1626 (Alonso y Zapatero 2000). Los avances son importantes en poco tiempo. Hay una transición gradual de nombres por regiones a nombres de las poblaciones de la costa, acompañado de un mejor perfil del contorno de la costa (Kjelbo 1966). La toponimia escandinava ya está españolizada en el XVI, excepto la remota costa del norte de Noruega, aunque el conocimiento de la región era aún muy confuso y no siempre se representa en la cartografía (MartínMerás 2000).

\section{Léxico de lugares en el texto de luan Scheffer}

Este texto pertenece a la época de pleno Barroco. El texto coincide con la etapa final de la época de esplendor de las letras españolas, entre 1568-1635. Según Abad Nebot (2008) en esta etapa se aceleran los cambios fónicos, se pasa de la pronunciación del castellano medieval a la pronunciación del español moderno.

Se trata de un documento oficial por el que se informa al rey español Felipe III acerca de los episodios de la intervención en Dinamarca de las tropas del Sacro Imperio en el período 1625-1629, en la Guerra de los Treinta Años. En el documento se relata la campaña de las tropas del Sacro Imperio que se enfrentan a las tropas del rey danés Christian IV.

Entre los rasgos léxicos del texto podemos destacar:

En este texto, del siglo XVII se utiliza la denominación Denemarca, lo que sugiere que ya estamos cerca de la denominación contemporánea, que es Dinamarca, con el paso de e $>\mathrm{i}$, y de e $>$ a. Emplea el nombre contemporáneo de Suecia. Por otra parte ya se ha fijado el nombre del mar interior como Báltico: "Las tierras del rey de Denemarca - desde la ciudad de Hamburg hasta el cabo de Schagen (hoy: Skagen), a la cual encierra los dos mares Balthico y Oceano".

Se utiliza todavía Septentrión - la palabra norte es neologismo.

Frisia es denominada Fridland, con la terminación land, que significa tierra. En el barroco se asiste a la desaparición de los grupos de consonantes, por lo que la conservación de la ' $\mathrm{d}$ ' intersilábica en Fridland, y la ' $\mathrm{h}$ ' de Balthico, pudiera ser interpretado como un rasgo decadente del Barroco. 
No se utiliza la W, sino que se dobla la $V$, como en el caso del General VvaIlenstein, Duque de Fridland, uno de los comandantes de las tropas del Sacro Imperio. También la emplea en Rio Vveser, y en Vviburg (Viborg).

Todavía en el siglo XVII no se tienen claros los límites geográficos del norte de Europa, pues el autor ubica a Escandinavia "en los confines de la tierra firme del Septentrion".

El texto es un ejemplo del aumento notabilísimo de topónimos (neologismos), pero el autor sigue una norma variable, utilizando criterios diversos e indefinidos para traducir los topónimos. A veces se inspira en la palabra arcaica que da origen al término moderno, como en el caso de Vensuffel (Vendsyssel?), que corersponde a una comarca al norte de la actual Ålborg, que Scheffer escribe Alborg, en el norte de Jutlandia "que los antiguos Ilamaron Cimbrica Chersonesus". Otras veces se guía por un sentido castellanizante, sin profundizar en el significado original de las palabras; por ejemplo Zunte (el restablecimiento de la -e final ya es un hecho), que es el Sund, el estrecho entre Dinamarca y Suecia, pero que en danés se dice Øresund- sund quiere decir estrecho y øre es una medida monetaria, en referencia al pago de aranceles para la navegación de entrada y salida al Báltico.

Otras veces, el autor adapta el término local, como en el caso de Zuder, que es posiblemente la ciudad de Thisted, en Norte de Jutlandia, que Scheffer denomina Nortjuland, por lo que sigue ahora la norma local, lo que también hace con el topónimo lutlandia (lo vocaliza, porque en español contemporáneo es Jutlandia). Desaparece la denominación de Dacia.

Los términos geográficos ya están modernizados en el XVII: ciudad, villa, provincia.

Preferencia de la c/z sobre la s en Celanda, que en danés moderno se escribe con S (Sjælland). El empleo de Copenagen muestra la influencia del alemán (Kopenhagen, frente al nativo København). Este nombre quiere decir puerto de comerciantes.

Tendencia latinizante podemos encontrar en los nombres Scania (Skåne) y Olada (Öland, que quiere decir 'Tierra de lagos').

En resumen, la tendencia a incorporar neologismos no sigue una norma fija. Como ilustra el escrito de este autor la traducción de los topónimos locales se realiza de acuerdo a criterios variables, algo propio del Barroco.

\section{Conclusión}

Desde la península ibérica Escandinavia se nombra a partir de los clásicos latinos y griegos. Los prejuicios culturales de la Edad Media dejaron su impronta en la manera de conceptuar a los paganos del norte. Es a partir de finales de la Edad Media cuando se comienzan a traducir los topónimos nórdicos de acuerdo a criterios lingüísticos que tienen como referencia la lengua del traductor. 
En estos dos textos hemos observado características de la lengua genuinas de cada periodo. A mi modo de ver, lo interesante de la comparación entre ambos es la manera que tiene cada autor de traducir el topónimo extranjero. Mientras que el autor anónimo del 'Libro del conocimiento', cuyas fuentes, en mi opinión, son los portulanos medievales, parece seguir un criterio castellanizante, en el sentido de adaptar el sonido de la palabra extranjera al sonido en casteIlano por medio de la grafía -y de manera arbitraria-, el autor del texto del XVII parece seguir unos criterios normativos, pero en consonancia con el espíritu del Barroco, varía de norma dentro del mismo texto. Ambos textos son reflejo de etapas distintas en el largo proceso de consolidación del idioma.

\section{Bibliografía}

ABAD NEBOT, F., Historia general de la lengua española. Valencia. Tirant lo Blanc. 2008.

ALONSO CASTELLANOS, F. y ZAPATERO LOURINHO, A., "How the Iberian School of Cartography and Geography spread through the North sea and the Baltic". En Enrique Martínez Ruiz y Magdalena de Pazzis Pi Corrales: Spain \& Sweden in the Baroque era (1600-1660). Fundación Berndt Wistedt. International congress Records. 2000. pp. 823-846.

BRAMSEN, B., Gamle danmarkskort. En historisk oversigt med biografiske noter for perioden 1570-1770. Rosenkilde og Bagger. 1975.

EKBLOM, R., "Ohthere's voyage from Skiringssal to Hedeby". Studia Neophilologica, 12:2, 1939, pp. 177-190.

JIMENEZ DE LA ESPADA, M., Edición del Libro del Conoscimento de reynos, tierras y señoríos. S. XIV. 1875.

JØRGENSEN, O., Alfred den Store Danmarks geografi. Odense universitetsforlag. 1983.

KEJLBO, I., Historisk kartografi. DHF. Købehavn. 1966.

LACARRA, M. J., LACARRA DUCAY, M. C. y MONTANER, A., Libro del conoscimiento de todos los rregnos et tierras et señoríos que son por el mundo et de las señales et armas que han. Edición facsimilar del manuscrito Z. Bayerische Staatsbibliothek. Institución Fernando el Católico. Excma. Diputación de Zaragoza. 1999.

LACARRA, M. J. y MONTANER, A., "Análisis codicológico y tradición del manuscrito Z". En Lacarra, Ducay y Montaner: Libro del conoscimiento de todos los rregnos et tierras et señoríos que son por el mundo et de las señales et armas que han. Edición facsimilar del manuscrito Z. Bayerische Staatsbibliothek. Institución Fernando el Católico. Excma. Diputación de Zaragoza. 1999.

LAPESA, R., Historia de la lengua española. Madrid. Escelier. 1942. 
LUND, A., Adam af Bremen krønike. Wormianium. 1990.

Mapa mondi, une carte du monde au XIVe siècle, l'atlas catalan, (1375). Bibliothèque Nationale de France; Montparnasse Multimedia. 1998.

MARINO, N., El libro del conoscimiento de todos los reinos. (The Book of Knowledge of All Kingdoms). Medieval and Renaissance texts and Studies. Vol. 198. Arizona Center for Medieval and Renaissance Studies. Tempe. Arizona. 1999.

MARTIN DE RIQUER, M., "La heráldica en el Libro del Conoscimiento y el problema de su datación". DICENDA. Cuadernos de filología hispánica. 6, 1987, pp. 313-319.

MARTIN-MERAS, L., "Un portulano español del norte de Europa". En Alberto Ramos Santana: Comercio y navegación entre España y Suecia. Siglos X-XX. 2000, pp. 91-103.

MONTANER, A. , "El libro del conoscimiento como libro de armería". En Lacarra, Ducay y Montaner: Libro del conoscimiento de todos los rregnos et tierras et señoríos que son por el mundo et de las señales et armas que han. Edición facsimilar del manuscrito Z. Bayerische Staatsbibliothek. Institución Fernando el Católico. Excma. Diputación de Zaragoza. 1999.

NORDENSKJOLD, A. E., Periplus: utkast till sjökortens och sjöböckernas äldsta historia: med talrika afbildningar af sjökort och kartor. Stockholm. 1987.

SANTIAGO, R., "La historia textual: Textos literarios y no literarios". En: Rafael Cano (ed.): Historia de la lengua española. Madrid. Ariel. 2004, pp. 533567.

SCHEFFER, I., Relación cierta i verdadera, sacada de avisos una y otra vez confirmados, de la rota $i$ huida del Rei de Denemarca, i total destrucció de su campo y exercito, i señaladas vittorias que la Sacra Magestad Cesare ha tenido aora nuevamente de sde 18 de octubre hasta fin de noviembre de 1627 en los Paises de la Saxonia inferior, i tierra firme del Septentrion. S. XVII. 